for tropical and subtropical regions.

Reviews of current knowledge about secondary production-microbial and faunal production in terrestrial, freshwater and marine ecosystems-were given jointly by $O$. W. Heal from Merlewood Research Station, England, and S. F. MacLean, from the University of Alaska, and also by A. Macfadyen, from the New University of Ulster. These reviews, which included discussion of the comparative productive efficiencies of different classes of organism and of different levels in the trophic chain, were necessarily painted with a coarse brush, as comprehensive data are rare for most types of ecosystem and much of the IBP research remains to be digested. Overall, there emerged estimates of the total secondary productivity in various types of ecosystem which seem secure to within a factor of ten. In particular, there was general agreement that we are beginning to understand the limits to the rate at which material can be processed as it passes along the food chain; but there can be major upsets and fluctuations from year to year (as, for example, in pest outbreaks or lemming cycles).

One of the more noteworthy conclusions of the studies of secondary production is that the bulk of it takes place below the ground. The birds and bees may engage scientific curiosity, but 90 to $99 \%$ of secondary production is by decomposer organisms in the soil and leaf litter. MacLean humorously underlined this point by remarking that if humans must consume at the secondary rather than exclusively at the primary level (eat meat instead of only plants), they should at least consider eating earthworms rather than beef.

In the plenary session devoted to "diversity, stability and maturity in natural ecosystems", G. H. Orians of the University of Washington, $\mathbf{R}$. $\mathbf{H}$. Whittaker of Cornell University and R. M. May of Princeton University marshalled various lines of empirical and theoretical evidence to suggest that communities with a rich array of species and a complex web of interactions (the tropical rainforest being the paradigm) are likely to be more fragile than relatively simple and robust temperate ecosystems. This inverts the conventional wisdom that "complexity begets stability", but seems more in accord with such reported facts as: (1) relatively simple arid ecosystems, including human populations with different economies, have persisted for long periods, fluctuating within well-defined limits (I. Nor-Meir, Hebrew University, Jerusalem); (2) estuarine ecosystems seem to be disturbed less by, and to recover faster from, indignities imposed by man than do marine ecosystems of higher diversity (D. F. Boesch, Virginia Institute of Marine Science); (3) the tropical rainforest seems to be a 'nonrenewable source' when it is disturbed over sufficiently large areas (A. GomezPompa and $\mathrm{C}$. Vázquez-Yanes, Institute of Biology, UNAM, Mexico).

The final two days centred around "diversity, stability and maturity in ecosystems influenced by human activities", and "strategies for management of natural and man-made ecosystems". Here most speakers agreed that many aspects of human activities are now on a scale comparable with natural biogeochemical processes, but that both short and long term impacts are uncertain. N. Alexander of Cornell University discussed the response of microbial communities to human activities. $\mathrm{He}$ pointed out that microorganisms are of enormous importance to the functioning of natural communities, that the impact of pesticides, polychlorinated biphenyls, acidic mine wastes and suchlike on various populations of microorganisms cause disturbances which range from "none to catastrophic", and that our ability to predict such outcomes are as yet woeful. In a major review paper, J. Jacobs of the University of Munich noted the ambiguities and uncertainties associated with the definition of human impact on

\section{Indigenous fish}

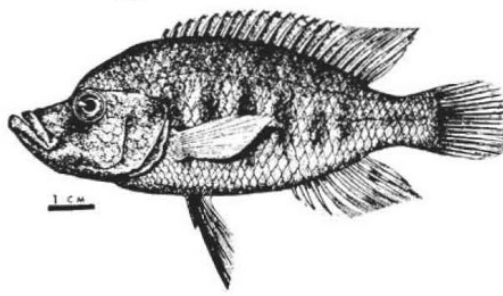

Haplochromis parorthostoma,

found only in Lake Victoria, East Africa. It is one of more than 100 endemic species of the cichlid genus Haplochromis which have evolved in the 750,000 years for which the lake has existed. According to P. H. Greenwood (author of Cichlid Fishes of Lake Victoria: The Biology and Evolution of a Species Flock; British Museum, London, 1974, £6.00) the explosive speciation was the result of a fortuitous combination of circumstances. Lake Victoria (originally a group of lakes) offered many environmental niches; the extent of anatomical specialisation of the ancestral river fish was neither too great nor too small to allow further specialisation in Lake Victoria; and the complexity of the fishes' breeding behaviour allowed barriers to interspecific crosses to evolve. natural systems, which are typified by a study of bird biomass and diversity in Finland: in Helsinki, there were 21 species and a biomass of (in some appropriate units) 1,089 ; in rural areas near houses, an average of 80 species and a biomass of 371 ; in areas undisturbed by man, an average of 54 species and a biomass of 297 .

The above account touches upon some of the larger themes of the congress. In so doing, it unfortunately has to pass over many interesting individual contributions. I particularly enjoyed the account by M. Cody, of UCLA, of the parallel and convergent evolution of the avifauna in 'Mediterranian' habitats in California, Chile, and Africa. This study was part of the IBP Convergent Ecosystems Project, and it provided detailed illumination of the way species with different evolutionary histories come to have similar roles, to provide striking constancy in the ecological structure of geographically separated communities. A diverse array of skills was brought to bear by G. H. Orians, R. G. Cates, D. F. Rhoades and J. C. Schultz, of the University of Washington, to elucidate some aspects of the cost-benefit accounting of the biochemical defences which plants maintain against the beasts which seek to eat them, and of the biochemical strategies in turn adopted by these consumers.

I think the congress would have been improved by widening its scope in two respeots.

By focusing on the structure and function of whole communities of plants and animals (community ecology). For instance, theoretical notions about domains of stability, or about some numerical characterisation of ecosystem 'resilience', are beginning to be brought into confrontation with the real world in recent studies of lowdimensional systems such as insect host-parasite populations; for complex multispecies communities I think such theoretical speculations are a long way from quantitative testing against nature.

Moreover, the congress was largely confined to technical aspects of how ecosystems work. Given that the word 'ecology' has come to mean all things to all people, such a narrow construction was probably the wisest course for a professional meeting. Even so, I would have liked to see one looser session, in which economists and professional ecologists aired speculations as to whether, for example, the contemporary global patterns of inflation are tied to real diminution in per capita supplies of food and energy, or whether these patterns are merely artifacts of present economic systems. To have held such a session may have run the risk of vulgarising the congress, but to have done otherwise runs the larger risk of seeming to fiddle while the world burns. 